\title{
Evaluación de aditivos químicos y biológicos en la vida de anaquel de la tortilla de nopal (opuntia ficus-indica)
}

\section{Evaluation of chemical and biological additives on the anaquel lifespan of nopal tortilla (opuntia ficus-indica)}

\author{
DOI: $10.46932 / \mathrm{sfjdv2n4-087}$
}

Received in: March 1st, 2021

Accepted in: May 30th, 2021

\section{Ceja León Edna Sofía}

Universidad de La Ciénega del Estado de Michoacán de Ocampo, Trayectoria de Genómica Alimentaria. Avenida Universidad 3000, Col. Lomas de la Universidad, C. P. 59103 Sahuayo, Michoacán, México

\section{Sánchez Saavedra Ma. Guadalupe}

Universidad de La Ciénega del Estado de Michoacán de Ocampo, Trayectoria de Genómica Alimentaria. Avenida Universidad 3000, Col. Lomas de la Universidad, C. P. 59103 Sahuayo, Michoacán, México

Autor de correspondencia: mgsanchez@ucemich.edu.mx

\section{Cárdenas Pérez Ricardo}

Universidad de La Ciénega del Estado de Michoacán de Ocampo, Trayectoria de Genómica Alimentaria. Avenida Universidad 3000, Col. Lomas de la Universidad, C. P. 59103 Sahuayo, Michoacán, México

\section{Loeza Lara Pedro Damian}

Universidad de La Ciénega del Estado de Michoacán de Ocampo, Trayectoria de Genómica Alimentaria. Avenida Universidad 3000, Col. Lomas de la Universidad, C. P. 59103 Sahuayo, Michoacán, México

\section{Núñez Oregel Verónica}

Universidad de La Ciénega del Estado de Michoacán de Ocampo, Trayectoria de Genómica Alimentaria. Avenida Universidad 3000, Col. Lomas de la Universidad, C. P. 59103 Sahuayo, Michoacán, México

\section{RESUMEN}

La tortilla de maíz (Zea mays L.), es la base de la alimentación mexicana, por su alto valor nutricional, no obstante, dado el nivel calórico que presenta se buscan alternativas que disminuyan este contenido. Una opción es adicionarle nopal, lo que disminuye el nivel calórico y proporciona beneficios importantes tales como, buena fuente de fibra dietética, actividad antioxidante, efecto prebiótico y con ello, es una alternativa para prevención de enfermedades crónicas degenerativas. Sin embargo, dada la alta actividad acuosa del nopal disminuye considerablemente la vida de anaquel de la tortilla, ocasionando deterioro significativo en pocos días. Para revertir tal efecto existen conservadores químicos como: el benzoato de sodio y ácido cítrico y conservadores biológicos como: las gomas arábigas, guar, xantana y el quitosano, que favorecen la baja actividad acuosa y permiten estabilizar de manera importante un alimento. Aunado al interés de la población de una disminución del consumo calórico también existe la preocupación de consumir alimentos libres de patógenos y la menor cantidad de aditivos posibles. Por lo anterior, en este trabajo se analizan diferentes conservadores químicos y biológicos con la finalidad de alargar la vida de 
anaquel de la tortilla de nopal con la mínima dosis posible. Para ello, a $1 \mathrm{~kg}$ de masa se agregó el agente conservante en diferentes concentraciones, se homogeneizó y posteriormente se elaboraron las tortillas a partir de $30 \mathrm{~g}$ de la masa, se dejó alcanzar la temperatura ambiente, empacaron y almacenaron a $25^{\circ} \mathrm{C}$ y $4{ }^{\circ} \mathrm{C}$, se registró diariamente el estado de la tortilla hasta observar cambios desfavorables. Se obtuvieron resultados con una duración de 5 días a temperatura ambiente con aditivos diferentes y de 25 y 37 días a temperatura de $4^{\circ} \mathrm{C}$.

Palabras clave: Tortilla de nopal, vida de anaquel, conservadores químicos y biológicos.

\section{ABSTRACT}

Corn tortilla (Zea mays L.) is the basis of Mexican food, due to its high nutritional value; however, given its high caloric content, alternatives are being sought to reduce this content. One option is to add nopal, which reduces the caloric level and provides important benefits such as a good source of dietary fiber, antioxidant activity, prebiotic effect and thus is an alternative for the prevention of chronic degenerative diseases. However, due to the high aqueous activity of nopal, the tortilla's shelf life decreases considerably, causing significant deterioration in a few days. To reverse this effect, there are chemical preservatives such as sodium benzoate and citric acid and biological preservatives such as gums arabic, guar, xanthan and chitosan, which favor the low water activity and can significantly stabilize a food. In addition to the population's interest in reducing caloric intake, there is also the concern of consuming food free of pathogens and the least amount of additives possible. Therefore, in this work, different chemical and biological preservatives are analyzed in order to extend the shelf life of nopal tortillas with the minimum possible dose. For this purpose, the preservative agent was added to $1 \mathrm{~kg}$ of dough in different concentrations, homogenized and then the tortillas were made from $30 \mathrm{~g}$ of dough, allowed to reach room temperature, packed and stored at $25{ }^{\circ} \mathrm{C}$ and $4{ }^{\circ} \mathrm{C}$, and the tortilla condition was recorded daily until unfavorable changes were observed. Results were obtained with a shelf life of 5 days at room temperature with different additives and 25 and 37 days at $4^{\circ} \mathrm{C}$.

Key words: Cactus tortilla, shelf life, chemical and biological preservatives.

\section{INTRODUCCIÓN}

Considerado un emblema para México, el nopal (Opuntia ficus-indica) es una especie de cactus perteneciente a la familia de las cactáceas, al orden Caryophyllales y al género Opuntia, que se caracteriza por crecer en zonas áridas, además de ser una especie perenne y suculenta, es decir, no pierde hojas en una estación del año y almacena gran cantidad de agua en sus tallos, además de que aporta grandes beneficios para la salud humana (Torres-Ponce, Morales-Corral, Ballinas-Casarrubias y NevárezMoorillón, 2015). Por otro lado la tortilla de maíz (Zea mays L.), es la base de la gran parte de la cultura alimentaria en el país por su alto valor nutricional; el promedio anual de consumo per cápita de tortilla hasta el 2011 fue de $78.5 \mathrm{~kg}$; sin embargo, aunque se considera alto, este ha disminuido debido a los cambios en los hábitos alimenticios de la población en los últimos 30 años. De acuerdo con la Unión Nacional de Industriales de Molinos y Tortillerías (UNIMTAC), el consumo en 2016 se ubicó en 110 kilos per cápita, un incremento de 34\% a los 82 kilos de 2012 (Vigía, 2017). No obstante, dado el contenido calórico que presenta se buscan alternativas que disminuyan este contenido. Una de ellas es la 
incorporación de nopal, sin embargo, su producción involucra una serie de problemáticas que van desde las económicas y de mercado, hasta las tecnológicas. En este último sentido, diversas empresas, entre ellas "Nopalsa", que se encuentra ubicada en la localidad de Emiliano Zapata, Municipio de Villamar, Michoacán, México, reporta graves problemas debido a la presencia de microorganismos contaminantes y probables patógenos, principalmente hongos, en dichas tortillas, los cuales reducen significativamente la vida de anaquel de estos productos. Para controlar la presencia de hongos en las tortillas de nopal podrían utilizarse diversas técnicas entre ellas el uso de conservadores químicos como el ácido cítrico y el benzoato de sodio, que actúan como antioxidantes, reducen el nivel de humedad de los alimentos, y evitan el crecimiento de microorganismos, en consecuencia ayudan a alargar la vida de anaquel. De la misma manera, también existen conservadores biológicos como goma xantana, goma arábiga, goma guar y quitosano, cuya función, entre otras, es la de atrapar el agua formando biopelículas protectoras, y tener actividad antimicrobiana, entre ellas la antifúngica, por lo que al ser adicionadas a un alimento podrían aumentar su tiempo de almacenamiento (Mora, 2013). Adicionalmente, no se conoce efecto perjudicial de la ingestión de estas sustancias para la salud humana (Calvo, 1991). Por lo anterior el propósito de este trabajo fue evaluar el efecto de diferentes aditivos químicos en la vida de anaquel de tortillas adicionadas con nopal (Opuntia ficus-indica) y establecer el efecto antifúngico de quitosano sobre Penicillium sp. Y determinar su efecto en la vida de anaquel de tortillas de $O$. ficus-indica.

\section{MATERIALES Y MÉTODOS}

\subsection{MATERIAL BIOLÓGICO}

El nopal fue proporcionado por la empresa "Nopalsa” ubicada en Emiliano Zapata, Michoacán, y trasportada al laboratorio de Tecnología de Alimentos de la Universidad de La Ciénaga del Estado de Michoacán de Ocampo. Los conservadores y aditivos fueron proporcionados por la misma casa de estudios.

Elaboración de la tortilla de nopal. Para la elaboración de la tortilla de nopal, se utilizó masa instantánea de maíz (MASECA) y nopal fresco en proporción 1:1. El nopal fue lavado y desinfectado con cloro (Cloralex) a una concentración de 20 ppm, se licuo durante 60 segundos y se incorporado a la masa homogeneizando manualmente. A partir de esta masa se tomaron las proporciones correspondientes para cada tratamiento.

Incorporación de aditivos químicos. Los aditivos o conservadores químicos utilizados fueron el benzoato de sodio y ácido cítrico en donde a cada uno se le realizó tres tratamientos con diferentes contracciones, para el benzoato de sodio se agregó 18, 9 y $4.5 \mathrm{mg}$ previamente disueltos en $100 \mathrm{~mL}$ de 
agua. Para el ácido cítrico se reutilizaron concentraciones de 6.7, 4.5, y $2.2 \mathrm{mg}$, disueltos de la misma forma que los tratamientos anteriores. Una vez establecidas y pesadas las dosis correspondientes, se incorporó a la masa y se homogeneizó, se elaboró la tortilla de $30 \mathrm{~g}$ cada una, posterior a ello, se colocaron en el comal a fuego medio, para su cocción. Se retiraron del fuego y colocaron sobre la mesa previamente desinfectadas hasta alcanzar temperatura ambiente para posterior a ello colocarlas sobre papel encerado y almacenar en charolas de plástico a $25^{\circ} \mathrm{C}$ y permitiendo el intercambio gaseoso. El mismo procedimiento se llevó a cabo para el ácido cítrico. Todo lo anterior se realizó por triplicado y considerando un testigo sin tratamiento.

Incorporación de aditivos biológicos. Los aditivos utilizados fueron goma Guar, goma xantana, goma arábiga y Quitosano. Las concentraciones utilizadas para las gomas fueron de 45, 22.5 y $9 \mathrm{mg}$ para cada una de ellas y de manera independiente. Respecto al Quitosano, se utilizaron dosis de 25 y $20 \mathrm{mg}$ previamente ajustado a un $\mathrm{pH}$ de 5.6 en vinagre al 5\%. Todo lo anterior se realizó por triplicado y considerando un testigo sin tratamiento (sin agregar el agente conservante). Una vez establecidas las dosis para los tratamientos de aditivos biológicos el procedimiento de elaboración y análisis de la tortilla se llevó a cabo de manera similar al del tratamiento de aditivos químicos.

\section{Comparación de aditivos químicos y biológicos en la vida de anaquel a $4{ }^{\circ} \mathrm{C}$.}

Se realizó un tercer experimento en donde se compararon los aditivos químicos y biológicos que en los experimentos anteriores mostraron mejores resultados, en este experimento se incluyó al Quitosano, dado que en experimentos preliminares in vitro mostró resultados positivos contra el hongo penicillium sp. El cual podría ser el principal causante de deterioro de la tortilla de nopal. En este experimento se consideró la mejor dosis de goma guar, goma xantana, benzoato de sodio y quitosano y se repitió el procedimiento de elaboración de la tortilla, pero ahora se almacenó en refrigeración $4{ }^{\circ} \mathrm{C}$. $\mathrm{Al}$ igual que en los experimentos anteriores se realizaron los tratamientos al menos por triplicado y considerando en todo momento un control sin el agente conservante. Finalmente los resultados fueron analizados con ayuda del programa R Project for Statistical, version 4.1.1 (Kick Things)

\section{RESULTADOS Y DISCUSIÓN DE RESULTADOS}

\subsection{EFECTO DE ADITIVOS QUIMICOS A $25^{\circ} \mathrm{C}$.}

De acuerdo con los resultados obtenidos el mejor tratamiento de aditivos químicos corresponde al benzoato de sodio a una concentración de $4.5 \mathrm{mg}$ y una vida de anaquel de la tortilla de nopal de 5 días a temperatura ambiente en comparación con el control que duró 3 días a la misma temperatura, este 
tratamiento no presentando diferencia significativa con el de 9 mg, también se observó (Figura 1) que el tratamiento de $18 \mathrm{mg}$ está muy cercano a los resultados de 4.5 y $9 \mathrm{mg}$, con una tendencia a disminuir la vida de anaquel de la tortilla, tales resultados podrían indicar que concentraciones superiores a los $18 \mathrm{mg}$ podrían no ser favorables para la conservación de la tortilla de nopal, sin embargo, si sería conveniente realizar otros ensayos con concentraciones menores a $4.5 \mathrm{mg}$ con la finalidad de encontrar la dosis minina que la preserve por más tiempo, tal como lo recomienda fennema, (2000). Lo anterior, concuerda con lo que señala fennema, (2000) y Luck y Jager, 1995, ya que menciona que el benzoato de sodio y sus sales tienen una acción antimicrobiana sobre el sistema enzimático de los microorganismos y específicamente posee un efecto inhibidor sobre el hongo penicillium spp; además de que a bajas concentraciones no se han reportado problemas a la salud. Sin embargo, algunos estudios realizados muestran que las personas prefieren alimentos que contienen aditivos naturales sobre aquellos que son preparados con aditivos sintéticos (Carocho et al., 2015) citado por López et al. (2021).

Con respecto al tratamiento de ácido cítrico no se encontró diferencia significativa con el control, lo cual indica que con las dosis analizadas no presenta ningún efecto para la conservación de la tortilla a $25^{\circ} \mathrm{C}$, en este caso también se podrían realizar más ensayos para ver qué efectos presenta con dosis superiores, sin rebasar la dosis máximas permisible de $0.5 \mathrm{~g} / \mathrm{kg}$ según la NOM-184-SSA1-202, también se podría combinar con otros conservadores tal como lo hicieron Saavedra et al. (2018), donde utilizaron el ácido cítrico como conservante alimenticio, y una mezcla entre el ácido cítrico con sorbato de potasio y el resultado fue significativamente notorio el conservante que le inhibió más fue el ácido cítrico con sorbato de potasio.

\subsection{EFECTOS DE ADITIVOS BIOLÓGICOS A $25^{\circ} \mathrm{C}$.}

En el ensayo con aditivos biológicos, los resultados arrojaron que el mejor tratamiento con una vida de anaquel de 6 días fue el de goma xantana a una concentración de $45 \mathrm{mg}$, no difiriendo estadísticamente con el tratamiento de goma aguar a una concentración de $9 \mathrm{mg}$, estos resultados indican que el tratamiento con goma xantana mientras mayor es la concentración mayor efectividad tiene, por lo que se podrían incrementar las dosis para ver si presenta mejores resultados, caso contrario a lo ocurrido en el tratamiento con goma guar que la menor concentración fue la que presento mayor eficiencia, lo que indica que por alguna razón dosis más altas inhiben el efecto antimicrobiano. De lo anterior es importante resaltar que una concentración de goma xantana debe ser 5 veces superior a la de la goma guar para obtener el mismo resultado, con respecto a lo anterior Gurkin, (2002) mostró que el uso de hidrocoloides en la producción de tortillas, prolongan la vida de anaquel además de disminuir la dureza de las mismas. Igual como menciona Yau et al. (1994) citado por Farías et al., (2002) la adición de gomas a las harinas 
nixtamalizada tiene como fin mejorar las propiedades de textura de la masa y tortilla, además de favorecer la retención de humedad, por lo que ayuda a no generar un medio óptimo para el desarrollo de microorganismos.

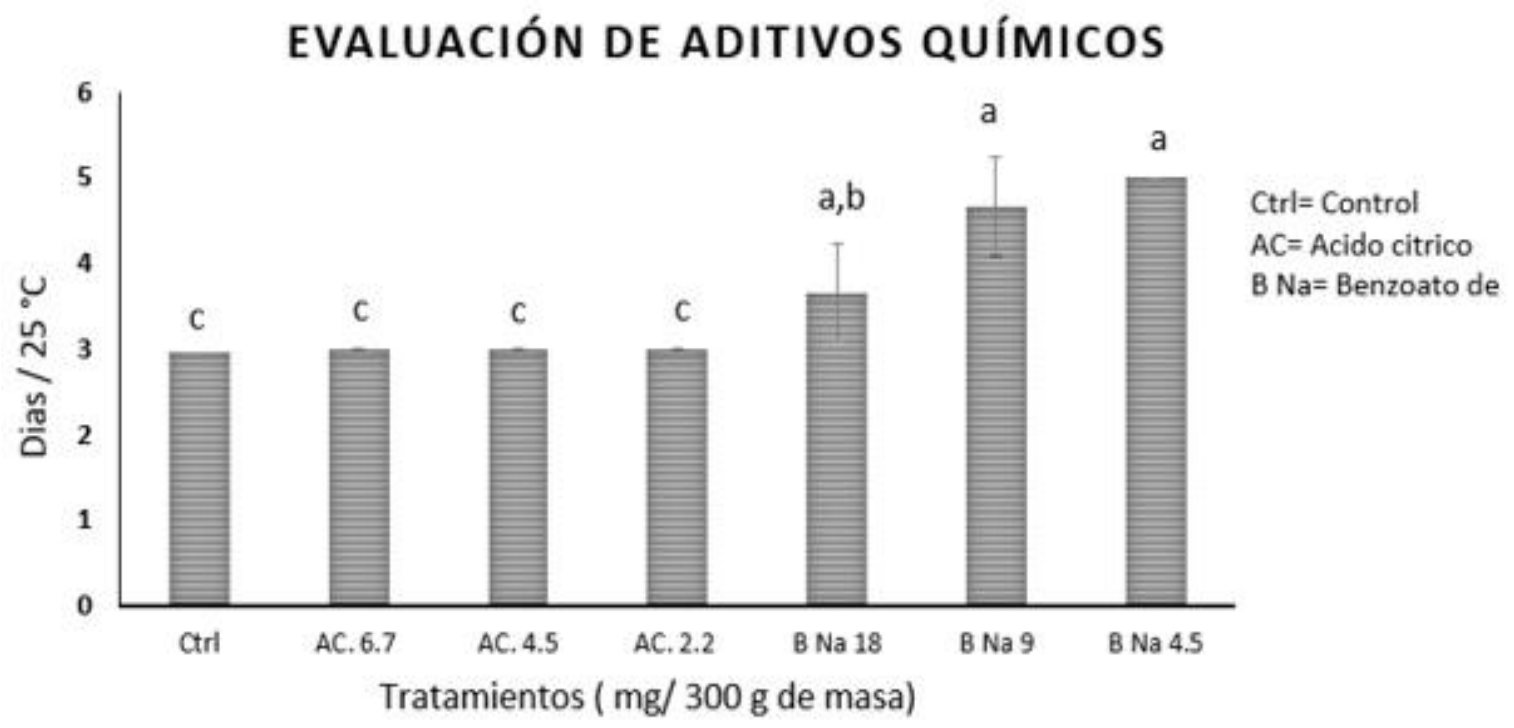

Figura 1. Evaluación de ácido cítrico y benzoato de sodio a $25^{\circ} \mathrm{C}$. Letras distintas denotan diferencia significativa entre tratamientos (ANOVA-Tukey) $p \leq 0.05, n=3$.

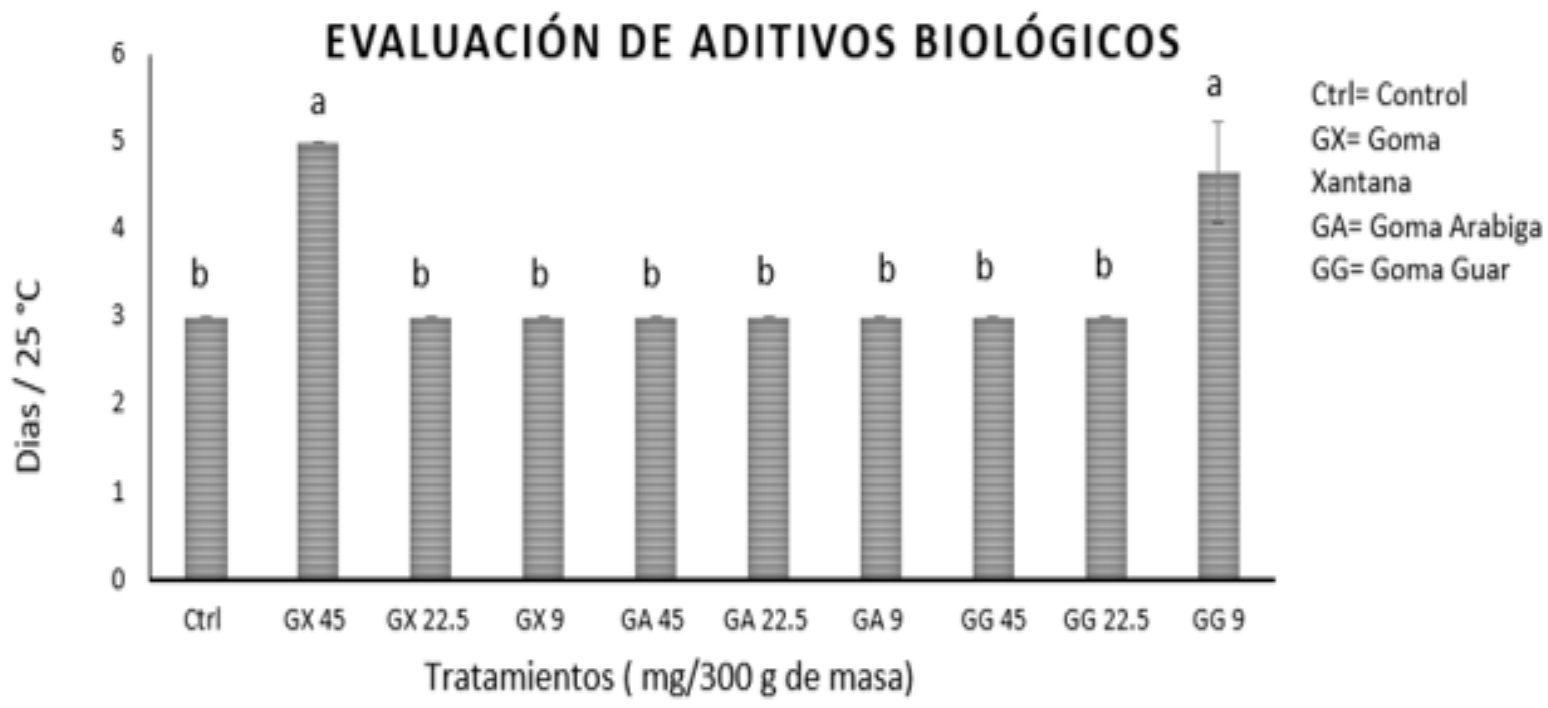

Figura 2. Evaluación de los aditivos goma guar, xantana y arábiga a $25^{\circ} \mathrm{C}$. Letras distintas denotan diferencia significativa entre tratamientos (Anova-Tukey) $p \leq 0.05, n=3$ 


\subsection{EVALUACIÓN DE LOS TRATAMIENTOS QUÍMICOS Y BIOLÓGICOS MÁS EFECTIVOS}

EN LA VIDA DE ANAQUEL DE LA TORTILLA DE NOPAL (OPUNTIA FICUS-INDICA) A UNA TEMPERATURA DE $4{ }^{\circ} \mathrm{C}$.

Como era de esperarse y dado que por sí sola la refrigeración es un método de conservación, los resultados fueron muy superiores al de temperatura ambiente, como se muestra en la fig. 3. Los mejores tratamientos fueron goma xantana con $45 \mathrm{mg}$, goma guar con $9 \mathrm{mg}$ y benzoato de sodio con $4.5 \mathrm{mg}$, con una vida de anaquel de hasta 37 días en comparación del control con una duración de 14 días, estos resultados están por encima de los mencionados por Sánchez y Vázquez (2018), ya que utilizaron goma Xantana en la tortilla de maíz y lograron alargar la vida de anaquel por dos semanas a una temperatura de $4{ }^{\circ} \mathrm{C}$. Lo anterior sugiere la importancia de mantener la cadena de frio en el tratamiento y conservación de la tortilla de nopal, Reyes-Pérez, et al. (2021) mencionan que cambio climático está ocasionando la modificación de las comunidades microbianas de cada zona con presencia de organismos que resisten mejor las nuevas condiciones, lo que afectará directamente la vida de anaquel de los alimentos.

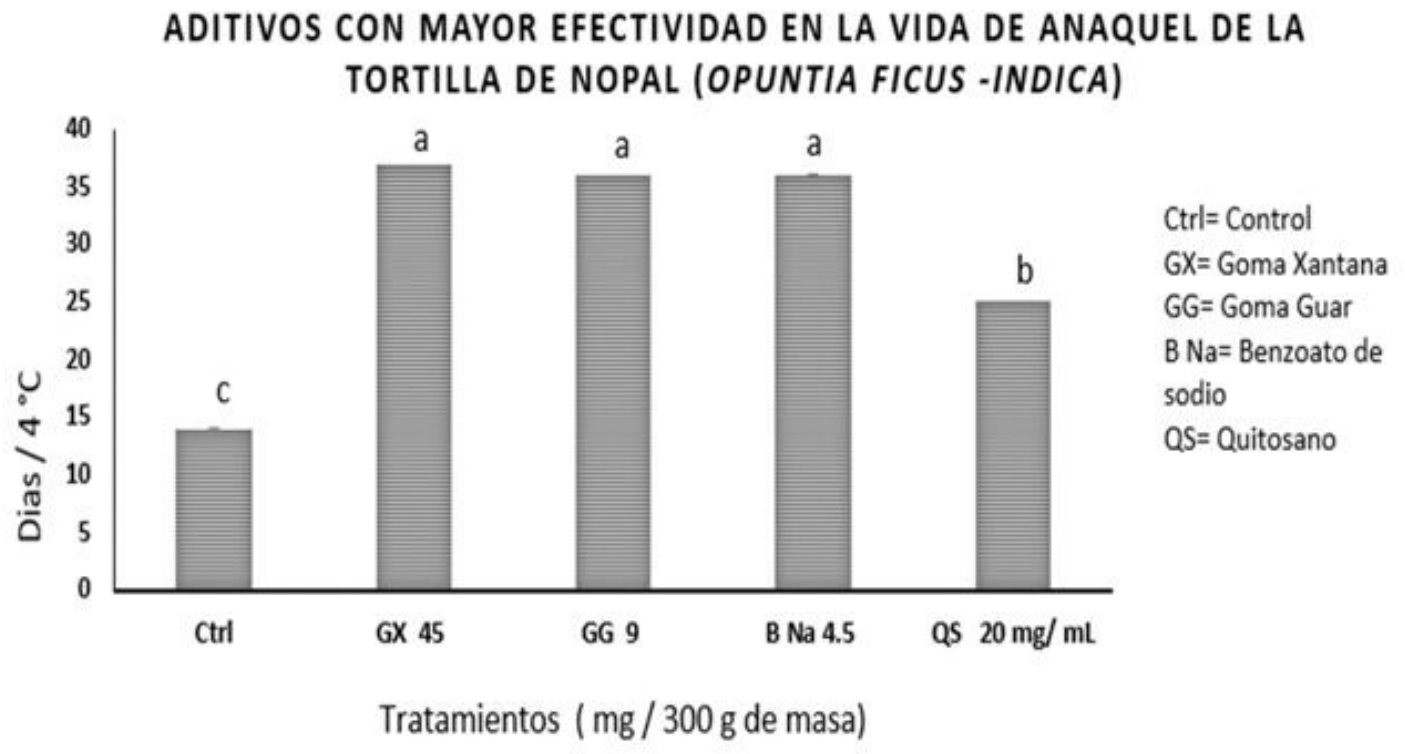

Figura 3. Evaluación de las mejores concentraciones de los tratamientos, benzoato de sodio, goma guar, goma xantana y quitosano a una temperatura de $4^{\circ} \mathrm{C}$. letras distintas denotan diferencia significativa entre tratamientos (ANOVA-Tukey) $p \leq 0.05, n=3$.

\section{CONCLUSIONES}

Los tratamientos de tortilla de nopal con benzoato de sodio, goma guar y goma xantana a $25^{\circ} \mathrm{C}$ con dosis de 4.5 .45 y $9 \mathrm{mg}$ respectivamente prolongaron la tortilla de nopal en todos los casos por 5 días, la misma tendencia se mostró en todos los conservadores a $4{ }^{\circ} \mathrm{C}$ pero aumentando de manera significativa la vida de anaquel de la misma por hasta 37 días. Lo cual indica que se pueden usar indistintamente cualquier de estos aditivos. Sin embargo, considerando la dosis utilizada y el tipo de 
conservador el que más se recomendaría usar sería el de goma guar por el ser un bioconservador, otra opción viable es el uso de quitosano, ya que logro una vida de anaquel de 26 días y al ser un bioconservador es probable una mejor aceptación ante los consumidores. En todos los casos son recomendables estudios más finos que den una mejor certeza de los resultados obtenidos. 


\section{REFERENCIAS BIBLIOGRÁFICAS}

C. Lárez-Velásquez (2006), quitina y Quitosano: materiales del pasado para el presente y el futuro. Avances en química, 1 (2), pp. 15-21.

C. Sáenz., H.Berger; J. Corrales-García., L. Galleti; V. García-Cortázar., I. Higuera., C. Modragón., A. Rodríguez-Félix; E. Sepúlveda., M.T. Varnero (2006), Utilización agroindustrial del nopal. Boletín de servicios agrícolas de la FAO, vol.162.

Lück, E., \& Jager, M. (1999). Conservación química de los alimentos: características, usos, efectos (No. 664.028 L939con2). Zaragoza, ES: Acribia.

Mendez Llorente, F., Ramirez Lozano, R. G., Lopez Carlos, M. A., Rodriguez-Frausto, H., Arechiga Flores, C. F., Bonilla-Salazar, A. \& Aguilera-Soto, J. I. (2011). Performance and nutrient digestion of lambs fed incremental levels of wild cactus (Opuntia leucotrichia). Journal of Applied Animal Research, 39(3), 248-251.

F. Owen. (2000).Ciencia de los alimentos (nutrition y salud). Editorial Limusa

Lück, E., \& Jager, M. (1995). Conservación química de los alimentos. Características, usos y efectos. Zaragoza, ES: Acribia.

Ipcdedios, (2013). Las plantas curativas de Dios. Wordpress.

Gurkin. (2002).Hydrocolloids ingredients that add flexibility to tortillas processing. Cereal Foods World, 47: 41-43

R.L. Torres-Ponce., D. Morales-Corral., M.L. Ballinas-Casarrubias y G.V. Nevárez-Moorillón. 2015. El nopal: planta del semidesierto con aplicaciones en farmacia, alimentos y nutrición animal. Revista Mexicana de Ciencias Agrícolas, vol. 6; pp. 1129-1142.

Reyes-Pérez, J. Moreno-Lara, J. Torres-Flores, D. Arrúa-Alvarenga, A. Quezada-Viay, M. 2021. Determinación de micotoxinas presentes en alimentos de consumo infantil comercializados en México. South Florida Journal of Development, Miami, v.2, n.4, p.5122-5134.

M. Ríos-Velázquez. (2017), Michoacán es el sexto estado líder en producción de nopal. Cambio de Michoacán.

S. Monrroy. (2018). Duelo nutricional, tortilla de maíz o tortilla de harina. El Universal.

D. Sánchez-Sandoval y L. Vázquez-Chávez. (2018).Vida de anaquel de tortillas de maíz nixtamalizado. Investigación y desarrollo en ciencia y Tecnología de Alimentos, 3: 17-22.

M. Calvon. (1991). Aditivos alimentarios: Propiedades, aplicaciones y efectos sobre la salud. Mira Ediciones. Zaragoza 